\title{
La Quete Musicale De L'authenticite : Un Transfert Sur L'inouï Ou Portrait Des «Baroqueux » En Aventuriers Du Timbre Perdu
}

\author{
Jean-Michel Vivès ${ }^{1}$
}

\section{RÉSUMÉ}

L'auteur de cet article s'attache à décrire les phénomènes psychiques qui ont présidé au mouvement baroque où des musiciens ont tenté de retrouver les sons qui étaient ceux qui avaient vu naître les œuvres interprétées. II se concentre sur la façon dont l'interprète tente d'approcher un son nouveau, non encore advenu, qu'il appelle le « transfert sur l'inouï ». Il y brosse un portrait des « baroqueux » en aventuriers d'un timbre perdu. Leur désir d'authenticité des interprètes serait celui de réentendre des timbres qui n'avaient plus cours. II approche les qualités de ce timbre à travers le parallèle entre André Schaeffner et Philippe Beaussant, et à travers eux entre les musiques africaines et l'aventure baroque : présence du bruit, du tremblé ou du trouble dans le son, qui aurait le pouvoir de convoquer au-delà de l'enveloppe sonore la dimension du réel.

Mots-ClÉs : baroque, musique, timbre, transfert sur l'inouï, voix.

\footnotetext{
${ }^{1}$ Professeur de Psychologie Clinique et Pathologique - Université Côte d'Azur, Membre du Comité Scientifique du Centre Culturel de Rencontre d'Ambronay. Psychanalyste - Toulon. Adresse de correspondance jeanmichelvives@gmail.com
} 


\section{JOUER BACH A SA MANIERE ?}

Le terme d'authenticité, qui fut rapidement associé au mouvement musical qui s'attacha à la redécouverte des œuvres issues du répertoire de la musique ancienne et à sa restitution dans les conditions qui auraient présidé à leurs créations, a très tôt été considéré comme suspect ${ }^{2}$. Ainsi, le chef d'orchestre Nikolaus Harnoncourt (19292016), qui a grandement participé à ce mouvement, put-il affirmer : «Des mots aussi inoffensifs qu' " authenticité » comportent une nuance négative ; on conteste a priori à ses partisans une pratique musicale engagée, voire, souvent, compétente » (Harnoncourt, 1984, p. 95). Comme on peut le deviner ici, " authentique ", pourrait assez vite virer à l'injure... Néanmoins, l'authenticité est une notion qui a orienté et servi de porte-drapeau à la redécouverte de la musique ancienne et à son interprétation. Ainsi, les valeureux pionniers pouvaient-ils prétendre être "authentiques" dans leur approche musicale face aux «réactionnaires » qui interprétaient essentiellement Bach et Haendel avec un orchestre pléthorique, un style hérité du XIXème siècle et sans recourir aux instruments qui avaient servi à faire entendre cette musique ${ }^{3}$ au moment où elle fut créée.

Tel est le cas de l'immense claveciniste Wanda Landowska (1879-1959) qui aurait affirmé: «Libre à vous de jouer Bach à votre manière ; moi je le joue à sa manière ».Cette affirmation peut aujourd'hui prêter à sourire. En effet, s'il est indéniable que Wanda Landowska (2005) a déployé en son temps d'importants efforts pour interpréter la musique de Bach selon des critères qui lui paraissaient relever dune réelle recherche d'authenticité ${ }^{4}$, nous sommes bien obligés de reconnaître, malgré toute l'admiration que nous pouvons lui porter, que sa manière d'interpréter Bach relève bien plus de sa manière à elle que de celle de Bach. Du moins telle que nous pouvons nous la représenter aujourd'hui à la lumière des travaux musicologiques de ces cinquante dernières années. Manière qui, sans aucun doute, sera encore bien différente dans cinquante ans, tant elle se trouve autant informée par les recherches

2 On a assez rapidement préféré parler d'interprétation historiquement informée plutôt que d'authenticité. L'idée d'historiquement informée étant plus relative et ouverte que celle d'authenticité.

3 Ainsi coexistent, au milieu des années 60, deux versions très différentes des Concertos Brandebourgeois de Jean-Sébastien Bach. L'improbable et presque déjà anachronique enregistrement effectué par Herbert Von Karajan en 1966 à la tête du somptueux orchestre philarmonique de Berlin pour la firme Deutsch Grammophon et celui réalisé par Nikolaus Harnoncourt à la tête du Concentus Musicus de Vienne composé d'instruments d'époque, deux ans plus tôt en 1964, pour la firme Teldec.

${ }^{4}$ Son ouvrage paru en 1909 au Mercure de France témoigne de la rigueur de sa démarche. 
savantes que par le « goût » d'une époque qui privilégie toujours certaines options au détriment d'autres.

Le choix que fit Wanda Landowska de son instrument, et que l'on n'ose à peine nommer aujourd'hui clavecin ${ }^{5}$ tant il s'éloigne des instruments que nous classons habituellement sous cette catégorie, témoigne suffisamment de cet état d'esprit. Ainsi pouvait-elle affirmer sans en être plus troublée que cela : « Je sais bien que la disposition des registres, sur les clavecins du temps de Bach, différait quelque peu 6 de celle de mon Pleyel. Mais que m'importe si afin, d'obtenir l'effet qui convient, j'use de moyens qui ne sont pas exactement ceux dont Bach pouvait disposer» (Landowska cité par Haskell, 1988, p. 99).

À partir de là que peut-on entendre par authenticité si l'on persiste à vouloir utiliser ce terme? Harry Haskell dans son très documenté ouvrage, Les voix d'un renouveau. La musique ancienne et son interprétation de Mendelssohn à nos jours, risque un début de définition, justement à partir de la phrase prêtée à Wanda Landowska. Ainsi propose-t-il : « En s'efforçant de jouer Bach comme il l'aurait fait luimême, Wanda Landowska a surtout réussi à le jouer à sa façon à elle. La fidélité à soi-même ne peut-elle être le début de l'authenticité ? » (Ibid., p. 305).

Si nous comprenons ce que l'auteur tente d'approcher ici à partir de cette délicate notion de « fidélité à soi-même » nous ne pouvons pas ne pas en repérer la dimension peu opératoire. Suffit-il d'être « fidèle à soi même » pour être sur la voie de l' « authenticité »? La réponse est non ! Karajan interprétant Bach en 1966 à la tête

\footnotetext{
${ }^{5}$ À ce sujet, Harnoncourt ne mâche pas ses mots : "On fabriqua des instruments à clavier de toutes dimensions et dans toutes les gammes de prix, qui étaient construits comme des pianos et dont les cordes étaient pincés par des plectres en cuir dur, et plus tard en différentes matières synthétiques. Ces instruments, on les baptisa " clavecins ", alors qu'il y avait entre eux et le clavecin à peu près la même différence de sonorité qu'entre un mauvais violon d'enfant et un Stradivarius. L'erreur passa inaperçue, car les critères faisaient défaut, puisque les musiciens ne savaient pas du tout comment un clavecin devait sonner ». Harnoncourt N. (1982) opus cité, p. 97.

${ }^{6} \mid l$ s'agit là d'un doux euphémisme. En effet, la firme Pleyel qui construisit un clavecin pour l'exposition universelle de Paris en 1889 s'inspira plus des pianos de concert que des instruments du XVIIle encore en l'état qui aurait pu servir de modèle. Le Grand modèle de concert utilisé par Wanda Landowska hérite de la technologie développée pour le piano (avec des cordes filées sous forte tension) et s'écarte résolument des clavecins historiques réalisés au cours des XVIle et XVIle siècles. L'aspect général est celui d'un piano à queue. La caisse est lourde et massive, sans fond et avec des renforts de section importante. La table d'harmonie est réalisée en contreplaqué de 3 couches de $2 \mathrm{~mm}$ chacune, en épicéa et son barrage est analogue à celui d'un piano. Cet instrument, pour lequel Manuel de Falla et Francis Poulenc composèrent des concertos qu'interpréta Wanda Landowska, possède des caractéristiques sonores si spécifiques qu'il serait bien aventureux de le remplacer aujourd'hui dans ces œuvres par une copie d'instrument du XVIIle. Toute la question de la singularité de l'articulation de l'instrument, du timbre qui lui est lié et de l'œuvre dans laquelle ils s'expriment est ici posée.
} 
de la philharmonie de Berlin est certes fidèle à lui-même, d'une cohérence absolue avec ce qui le constitue en tant que musicien (beau son, dynamiques et phrasés « romantiques », parfaite justesse d'intonation...), pour autant lorsque l'on écoute aujourd'hui son bel mais étrange enregistrement des Concertos Brandebourgeois on se persuade assez aisément qu'il ne saurait être considéré comme « authentique ».Bach reconnaitrait-il son œuvre ainsi ? II est peu probable que cela soit le cas. «La fidélité à soi-même » ne saurait donc être le critère qui permet de juger d'une démarche visant une interprétation «authentique ».

Un autre mécanisme, premier et plus subjectif, me semble nécessaire. Mécanisme qui donne son cachet « d'authenticité » à la démarche de Nikolaus Harnoncourt en 1964 malgré les défauts qui ne peuvent pas ne pas nous sauter aux oreilles plus de cinquante ans plus tard, comme par exemple la justesse d'intonation parfois aléatoire qui faisait dire à Jean-Luc Macia dans une discographie comparative des Concertos Brandebourgeois de Bach parue dans la revue Diapason de novembre 1998 : «cette version reste unique pour sa charge émotionnelle et sa valeur historique plus que pour ses qualités intrinsèques ». Ce qui fut unanimement reconnu et salué et qui divisa les auditeurs est le choc lié aux couleurs et donc aux timbres de l'orchestre utilisé. Ce qui oriente Harnoncourt semble être une mise en avant des instruments originaux même s'ils peuvent surprendre, voire déranger ou choquer l'écoute de l'auditeur. Cette démarche est ce que je propose de nommer : le transfert sur l'inouï (Vivès, 2012). Inouï qui relèverait d'un son non encore advenu, non totalement stabilisé mais que l'interprète tente d'approcher à l'occasion de son interprétation. Inouï que je propose de penser à partir de la notion de timbre. Ainsi, Wanda Landowska, malgré un instrument assez éloigné des clavecins historiques, et Nikolaus Harnoncourt, malgré des instruments historiques parfois encore incomplètement maîtrisés, visent un son au-delà de ce qui est qui immédiatement offert à l'oreille. Certes le résultat n'est pas parfait ${ }^{7}$ et Nikolaus Harnoncourt est un musicien bien trop accompli pour ne pas l'avoir su. Pourquoi s'en satisfit-il alors ? Parce ce que ce qu'il visait se situait au-delà de ce qui s'entendait. Là où Karajan s'attachait au rendu sonore immédiat quasi parfait pour le plus grand plaisir de nos oreilles, Landowska et Harnoncourt se risquaient à viser un au-delà du principe de plaisir musical et se

\footnotetext{
${ }^{7}$ Ce n'est plus le cas aujourd'hui. La facture des instruments anciens et les instrumentistes qui en jouent ont fait d'énormes progrès en plus de 50 ans et un orchestre " baroque ", depuis bien longtemps, ne rencontre plus ce type de difficultés.
} 
révélaient orientés par lui. Non un son beau mais un son « juste » jusque dans ses erreurs d'intonation. Ce qui fait qu'aujourd'hui encore, les enregistrements de Landowska et les premières gravures de Harnoncourt ne se réduisent pas à de simples témoignages de l'évolution de l'interprétation de la musique ancienne c'est que nous y percevons cette recherche, certes balbutiante mais assurée, d'un son inouï à partir de la restitution de timbres qui orientaient et mettaient en forme leur quête. Harnoncourt me semble approcher cela lorsqu'il affirmait : « II s'agit donc de savoir pourquoi un musicien se décide pour tel ou tel médium sonore » (Harnoncourt, 1984, p. 98). Après tout c'est bien au sujet de ces timbres qui étaient à nouveau entendus après plus d'une centaine d'années de silence, que détracteurs et admirateurs enthousiastes se déchireront.

\section{Le Timbre: Dimension Reelle Du Son Musical}

Nous devons ici, pour préciser notre hypothèse, définir plus avant ce que nous entendons par timbre, cette notion étant loin de faire l'unanimité ${ }^{8}$. Pour cela, rappelons rapidement quels sont les paramètres musicaux du son :

- La hauteur (un son grave / aigu) mesurable en Hertz qui en détermine la fréquence.

- La durée (un son court / long) mesurable en secondes.

- Le volume, son intensité (un son doux / fort) mesurable en décibels.

- Le timbre qui, selon la définition d'Émile Littré, est « le caractère d'un son indépendamment de son rang dans l'échelle, caractère tenant à des sons harmoniques qui coexistent avec le son fondamental et qui lui forment une espèce d'accompagnement; cette espèce d'accompagnement, dont l'oreille ne discerne pas les éléments, est précisément le timbre ».

Le dernier paramètre, le timbre, est le plus énigmatique et le plus difficilement cernable des quatre ${ }^{9}$. Contrairement à la hauteur, la durée et l'intensité, il ne se mesure

\footnotetext{
${ }^{8}$ Makis Solomos (2013) a effectué un relevé précis de l'évolution de la notion de timbre et des difficultés qui sont associées à son utilisation.

9 Jean-Jacques Rousseau qui semble avoir été le premier à avoir officialisé le mot « timbre » dans son acceptation moderne avait très justement repéré cette limite. Ainsi affirme-t-il dès 1767 dans son Dictionnaire de musique : "Cependant personne que je sache n'a examiné le son dans cette partie (le
} 
pas et échappe donc à toute possibilité d'être totalement cerné et défini. «On ne saurait d'un seul mot le définir », affirme à propos du timbre le compositeur Charles Koeclin (1867-1950) à l'occasion de son Traité d'orchestration (Koeclin, 1944, p. 12). Il est pourtant, et c'est là un de ses paradoxes, immédiatement identifiable. À tel point que le timbre est pour la voix d'une personne l'équivalent des empreintes digitales, on parle d'ailleurs d'empreinte vocale.

Ainsi, le timbre est ce qui fait qu'un son produit à la même hauteur, de même durée, avec la même intensité, ne ressemble pas à un autre, créant ainsi une vibration unique et singulière. Le timbre caractérise ce qu'on appelle aussi la «couleur» du son. Celle-ci n'est jamais pure, mais résulte d'un enchevêtrement complexe dans lequel d'autres fréquences sonores (harmoniques, réverbérations) viennent se greffer sur la fréquence initiale. Le timbre dépend aussi du « contour temporel » du son (attaque, chute, tenue, extinction). On ne saurait effectuer la mesure d'un timbre (il n'existe pas d'unité de mesure du timbre), mais on peut afficher son spectre sonore à l'aide d'analyseurs qui identifient et permettent de visualiser les diverses fréquences qui lui sont associées. Deux sons peuvent donc avoir la même hauteur et la même puissance, ils ne peuvent avoir le même timbre.

Le timbre n'est pas connaissable par la mesure directe mais il est par contre reconnaissable. On ne connait pas un timbre, on le reconnait. À partir de là, nous serons condamnés pour parler du timbre à utiliser des termes qui introduisent une comparaison avec d'autre sens : un timbre peut être doux ou dur, voilé ou éclatant... On comprend que ce détour obligé par les analogies et autres métaphores pour tenter, vainement, de cerner un timbre puisse indisposer ceux qui voudraient que le rapport au musical puisse s'effectuer sans reste, évacuant ce qui de la musique ne pourrait se dire.

À partir des éléments développés ci-dessus qui montrent que le timbre est ce qui échappe au pouvoir de symbolisation en ce qu'il reste non immédiatement traduisible en chiffres, mesures ou mots nous pouvons affirmer qu'il constitue la dimension réelle de l'instrument ou de la voix qui le produit. Le réel, pour la théorie

timbre) ; laquelle (...) se trouvera peut-être avoir des difficultés : car la qualité du timbre ne peut dépendre, ni du nombre des vibrations, qui fait le degré du grave à l'aigu, ni de la grandeur ou de la force de ces mêmes vibrations, qui fait le degré du fort au faible. II faudra donc trouver dans le corps sonore une troisième cause différente de ces deux pour expliquer cette troisième qualité du son et ses différences ; ce qui, peut être, n'est pas trop aisé ». Cité par Makis Solomos (2013, p. 26. C'est nous qui soulignons). 
psychanalytique, est cette dimension du monde qui vient imposer une limite au savoir, limite à partir de laquelle il ne peut être appréhendé mais plutôt cerné et déduit ; le réel dans sa globalité et sa complexité c'est l'impossible à dire. Le timbre relève bien de cette dimension d'impossible: les mots peuvent tenter de l'approcher mais ne réussissent jamais à le saisir. Je fais l'hypothèse que les pionniers de la musique ancienne furent ceux qui avaient le désir d'entendre ce qui n'avait plus été entendu : un violon, une voix, un hautbois dont les timbres n'étaient pas ceux qui s'entendaient alors.

Car c'est sur cette dimension réelle que l'instrumentiste et l'auditeur vont accrocher leur désir d'entendre ce son ${ }^{10}$ et non un autre, même si ce son là n'est pas le plus abouti au moment où il est retrouvé et produit. Ce désir de timbre trouve à s'illustrer d'une façon éclatante chez les lyricomanes qui élisent la voix de telle ou telle chanteuse comme étant celle qui les fera préférentiellement vibrer, voire résonner jusqu'à l'extase ${ }^{11}$. Une illustration de cette mélomanie ${ }^{12}$ pourrait être trouvée dans les empoignades opposant les admirateurs de Maria Callas à ceux de Renata Tebaldi qui sont restées célèbres. La recherche effrénée d'un timbre élu par l'amateur comme étant le lieu d'où " ça » l'appelle, laisse supposer que ce dernier y entende une réponse. L'amateur y perçoit son objet et tente alors de s'en approcher, voire de s'en emparer $^{13} \ldots$

\footnotetext{
${ }^{10}$ C'est sur cette question du timbre que se fait également, je crois, le choix d'un instrument de musique chez un enfant qui ne connaît pas encore tout l'étendue de l'instrumentarium à sa disposition. Ainsi ma fille, alors âgée de 5 ans, entendant à la radio le concerto pour clarinette de Mozart me demanda le nom de l'instrument qui jouait et affirma avec assurance que c'est de la clarinette qu'elle jouerait. Ce qu'elle fit. On le voit ici c'est un timbre s'exprimant à l'occasion d'un chef d'œuvre le mettant en jeu qui fait appel ici. C'est ce que l'on pourrait appeler le transfert sur le réel du timbre, ou l'inouï.
}

11 Une scène du Farinelli (1994) de Gérard Corbiau illustre avec une grande justesse l'effet du timbre sur le corps de l'auditeur. Le célèbre castrat commence un air et aperçoit une auditrice distraite en train de feuilleter un livre et de boire du thé dans sa loge. II interrompt l'orchestre et commence à enfler un son qui semble devoir ne jamais finir. La jeune femme devient attentive, palpite, rosit et finalement se rend... extase et jouissance lyriques liées non à une mélodie ou un rythme. II ne s'agit là que d'une note tenue, c'est donc bien un timbre qui vient toucher l'auditrice et la faire défaillir.

${ }^{12}$ La langue grecque possède un certain nombre de composés en -manês (adjectifs) et -mania (noms), qui renvoient à diverses formes de folies ou de passions : le mélomane est, étymologiquement, fou de musique (mélos). La langue n'a pas choisi d'en faire un amoureux de la musique, un " mélophile ", mais un maniaque, un possédé... Disons que nos valeureux conquistadors, partis à la recherche du timbre perdu, étaient possédés par cette question. Ainsi pourrions-nous nous risquer à dire qu'ils étaient délicieusement timbrés. C'est cette notion de timbre qui, j'en fais l'hypothèse, permet de répondre à la question de Nikolaus Harnoncourt citée plus haut : - "Pourquoi un musicien se décide pour tel ou tel médium sonore ?» Parce que, j'en fais l'hypothèse, il veut entendre et faire entendre ce timbre et pas un autre.

13 Comme nous pouvons le voir dans le film Diva (1981) de Jean-Jacques Beinex où Jules, un jeune postier, est fasciné par une célèbre diva, Cynthia Hawkins, qui n'a jamais consenti à faire enregistrer sa 
Cette notion de timbre est pourtant tellement problématique que certains auteurs sont allés jusqu'à proposer de s'en passer. Ainsi Michel Chion n'hésite-t-il pas à affirmer : «D'autres (notions) doivent être abandonnées, puisqu'elles s'avèrent, derrière leur fausse évidence, des notions causalistes inaptes à qualifier le son. C'est le cas du timbre. Après Schaeffer, il faut donc redire que la notion de «timbre » toujours enseignée comme scientifiquement valable est un amalgame sans consistance de données diverses »(Chion, 2018, p. 184). La charge est sévère et il ne s'agit là nullement d'une provocation de la part de cet auteur qui a si justement étudié la dynamique du son mais plutôt de la mise en évidence des limites de cette notion pour rendre compte des développements contemporains de la musique. Néanmoins, pour la période qui nous intéresse cette notion reste indépassable. C'est bien pour faire résonner des timbres spécifiques que Monteverdi indiqua précisément sur la page de garde de la partition de l'Orfeo la composition de l'orchestre et que Bach composa ses Concertos Brandebourgeois.

\section{« Le Bruit Meme Que Cette Musique Faisait »}

Cette hypothèse d'un transfert sur le timbre peut être déjà repérée chez Philippe Beaussant qui il y a trente ans, alors que le mouvement «baroque » commençait à s'imposer avec force dans les salles de concert, les festivals et les maisons d'opéra ${ }^{14}$ n'hésitait pas à affirmer : « qu'il (est) possible de communiquer avec la musique d'une époque non pas seulement par les formes qu'elle nous avait transmise, mais à travers le bruit même que cette musique faisait... » (Beaussant, 1994, p. 18. Les italiques sont de l'auteur). Il est intéressant que Philippe Beaussant utilise le terme de bruit qui nous renvoie ici à la matière sonore pouvant se situer du côté de ce qui échappe à la mesure, justement là où nous pourrions situer le timbre. En effet, comme l'a parfaitement montré Michel Chion (2018, p. 71-79), l'instrument qui produit une note

\footnotetext{
voix. Lors d'un concert parisien au théâtre des Bouffes-du-Nord, Jules enregistre clandestinement son récital. Après avoir reçu un autographe de la diva, il vole sa robe en coulisses puis s'enfuit. Sans le savoir, Jules rentre également en possession d'un autre enregistrement recherché lui, par des malfrats transformant la quête de la voix en dangereuse course poursuite. Le roman de Jules Verne Le château des Carpathes (1889) traite lui aussi de cette possession par et de la voix.

14 Le choc esthétique lié à la représentation de l'opéra de Lully, Atys, en 1987 dans une mise en scène de Jean-Marie Villégier et dirigée par William Christie à la tête des Arts florissants (représentation qui a remporté un immense succès auprès du public qu'il était difficile de prévoir) marque d'une certaine manière la victoire du camp baroque. La musique ancienne n'est plus confinée dans quelques festivals spécialisés et périphériques mais désormais attire un public large qui s'enthousiasme pour une œuvre qui obéit à des codes qui n'ont rien à voir avec ceux de l'opéra du XIXème et pourtant réussit à les toucher et les convaincre par delà les 300 ans qui les séparent de la création de l'œuvre.
} 
inscrite sur la partition produit également des bruits parasites qui, s'ils ne sont pas notés sur la partition, appartiennent en propre à la production sonore musicale : cliquetis de clés pour la flute ou la clarinette, glissés aigus créés par le déplacement des doigts sur les cordes de la guitare, grondement indistinct de l'extrême grave de la contrebasse ou de l'orgue, frottement de l'archet sur les cordes du violon... Tous ces bruits pouvant être perçus comme parasites mais qui participent activement à la constitution du timbre de l'instrument. À partir de là, nous pourrions dire que la musique s'érige sur et contre le bruit qui en retour la hante pour toujours à travers le timbre, l'obligeant à payer ainsi son tribut au réel.

André Schaeffner (1895-1980), important anthropologue et ethnomusicologue français qui a dirigé le département d'ethnomusicologie du Musée de l'Homme a donné une intéressante illustration de ce rapport entre musique et bruit dans son ouvrage Les origines des instruments de musique. II y cite Gide, qui avait repéré à l'occasion de son voyage au Tchad combien les musiciens de ce pays « avaient horreur du son net, besoin de le troubler et de noyer son contour » (Schaeffner, 1994, p. 52) par l'ajout et l'entremêlement de corps sonores où la vibration de l'un entraîne celle de l'autre. Le son ainsi produit n'est plus pur mais intègre une dimension de bruit aléatoire de grésillement de son contour qui en fait également l'intérêt. André Schaeffner va plus loin et rapproche cela, assez étonnamment à première vue, des ornements ou agréments dans la musique française de clavecin des XVIle et XVIIle siècles. Agréments nommés mordant, pincé, trémolo, flattement par quoi, selon la magnifique expression de Schaeffner, « un vieux désir de bruit trouve encore à s'exaspérer auprès du pur « contour » des sons » ( (lbid. p. 53). Les pionniers du mouvement baroque en s'attachant aux instruments qui avaient été ceux utilisés par les compositeurs seraient ceux qui n'auraient pas reculés horrifiés devant ce « vieux désir de bruit $\gg 15$.

\footnotetext{
15 Une petite fille de mon entourage, un jour qu'elle assistait au récital d'une célèbre claveciniste amie de ses parents, se vit poser par la concertiste la question suivante : "Aimes-tu le clavecin ? ». Ce à quoi la petite répondit : “Oui, mais c'est un petit peu moche.... ». Cette petite fille devenue elle-même musicienne aujourd'hui entendait sans doute « ce vieux désir de bruit » qui hante le clavecin et que l'auditeur acculturé refoule en s'attachant au « pur contour des sons ». II est amusant de repérer que Rousseau pouvait, à l'instar de cette petite fille, affirmer : "II y a même des instruments, tel que le clavecin, qui sont à la fois sourds et aigres ; et c'est le plus mauvais timbre ». Cité par Makis Solomos (2013) opus cité, p. 26.
} 
Philippe Beaussant insiste dans cette direction et précise : «Toutes ces questions (diapason, tempérament, notes inégales...) sont importantes, et on doit en parler : mais elles ne sont que techniques. Elles n'ont de signification que dans la perspective, qui seule est fondamentale, d'un souci, d'une recherche du son, de la couleur du son, de la vérité du son, de la vérité sonore d'une œuvre. Ce qui a orienté et dirigé le rapport nouveau qui s'est établi entre les interprètes et la musique ancienne, ce n'est ni la nostalgie du $415^{16}$, ni le culte des voies blanches, ni le rituel des notes pointées, ni même fondamentalement la volonté de retrouver l'authenticité historique. C'est que certains soient partis « à la recherche du son perdu », et d'autres pas. La césure se situe exactement en ce point. » (Beaussant, 1994, p. 19. Les italiques sont de l'auteur).

Traduit dans les termes qui sont les nôtres cela deviendrait : les « baroqueux » sont ceux qui ont transféré sur cet inouï qu'était le timbre d'instruments et de voix oubliés, voire rejetés et qui se sont laissés guider par ce réel qui tout en échappant donnait une direction à leur démarche.

II est ainsi intéressant de repérer qu'un des moments essentiels de ce mouvement de « reconquête » fut le « transfert » de la voix de contre-ténor de la scène religieuse (le chœur) à la scène profane où il devint à proprement parler le porte-voix du renouveau musical au cours des années 1940 et 1950. Le chanteur qui assura ce transfert est Alfred Deller (1912-1979). En rendant dans ses concerts et ses enregistrements la sonorité, le timbre oublié, car « fondu » dans les maîtrises et les chœurs anglais où il existait encore, Alfred Deller proposait un inouï au sens propre du terme, un non encore entendu.

Cette révélation ne fut pas sans conséquences et Philippe Beaussant s'amuse des transports horrifiés de certains critiques à l'écoute de cette voix interprétant l'Ode à Sainte Cécile de Purcell : « J'ai souvenir (...) des transports indignés, des furieuses altercations, qui faisaient vibrer mon poste de radio lorsque s'élevait la voix rocailleuse d'Antoine Golea : « C'est indécent... c'est obscène... Je ne peux supporrrter ces sons andrrrogynes... »(Beaussant, 1994, p. 48).

Mais quelles furent donc la spécificité et la charge, intolérables pour certains, de la démarche de Alfred Deller? Non seulement il ressuscitait la musique

\footnotetext{
16 Hauteur du diapason préférentiellement utilisée par les instrumentistes baroques mais pouvant également fluctuer alors que le diapason contemporain est stabilisé à $440 \mathrm{~Hz}$. Le diapason contemporain (440) serait plus haut d'un demi ton que le diapason « baroque » (415).
} 
élisabéthaine mais il le faisait en lui rendant le timbre qui lui était indissolublement lié ${ }^{17}$. Le compositeur britannique Michael Tipett (1905-1998) ne s'y est pas trompé lorsqu'il affirme que la voix de Deller faisait remonter les siècles à rebours. L'importance de la redécouverte du timbre de contre-ténor va dans le sens de l'affirmation de Arnold Dolmetsch (1858-1940), musicologue franco-suisse naturalisé britannique, violoniste et facteur d'instruments qui toute sa vie œuvra pour la réhabilitation de la musique ancienne avec des «pratiques authentiques » et qui dès 1930 affirmait sans ambages : « l'étude de la musique de quelque période qu'elle provienne (...) (devrait) commencer par celle des instruments de ladite période » (Cité par Haskell, 2013, p.295). Nous retrouvons ici l'idée d'une nécessaire négociation entre l'instrumentiste, l'instrument adéquat et le répertoire interprété. Négociation qui implique des pertes pour qu'elle puisse aboutir et qui spécifiera la quête de l'authenticité musicale. Ainsi, si nous revenons à l'enregistrement des Concertos Brandebourgeois effectué par Harnoncourt avec le Concentus Musicus de Vienne en 1964, il est évident que le chef accepte dans la négociation qu'il effectue à l'occasion de cette gravure de perdre la sureté d'intonation et parfois la justesse pour gagner en richesse de couleurs et de timbres des instruments d'époque. C'est l'inouï des timbres des instruments du XVIIlème siècle qui guide son travail. Harnoncourt pose d'ailleurs la question en ces termes: «Suis-je prêt, pour telle ou telle « conquête », à payer le prix inscrit dans la nature même de la chose ? À renoncer par exemple, pour gagner en puissance, aux nuances et aux couleurs subtiles ainsi qu'à l'aisance technique (piano) ; ou à acheter l'égalité parfaite sur le plan de la dynamique et de l'intonation de tous les demi-tons utilisables en perdant l'intonation spécifique de chaque tonalité et le timbre individuel de pratiquement chaque note (flûte, entre autres) ? On pourrait citer des exemples de ce genre pour chaque instrument ou presque. Le plus souvent, en effet, fasciné à chaque fois par l'« amélioration » obtenue, on n'a pas remarqué au premier abord qu'il fallait, en même temps, sacrifier quelque chose, ni, à plus forte raison, l'objet de ce sacrifice. » (Harnoncourt, 1984, p. 91).

\footnotetext{
17 Philippe Herreweghe a poussé cette recherche dans ses conséquences pratiques les plus abouties en choisissant de créer ou d'emprunter les phalanges lui permettant de disposer du groupe vocal ou musical le plus en adéquation pour chacun des répertoires qu'il aborde. Le Collegium Vocal pour interpréter le baroque vocal allemand. La Chapelle royale pour le baroque français. L'orchestre des Champs-Élysées, créé en 1991, pour interpréter le répertoire classique puis romantique. L'éphémère ensemble vocal Européen servit à l'interprétation des polyphonies de la renaissance et enfin l'utilisation de l'Ensemble Musique Oblique pour interpréter la musique contemporaine.
} 
Cette quête du son n'est pas spécifique à la démarche baroque - même si elle y est particulièrement active - et Philippe Beaussant en 2001, à l'occasion d'un entretien, le relève avec acuité : « Je pourrais conclure en ajoutant que le phénomène baroque de ces dernières années n'est pas isolé. II est parallèle, dans d'autres domaines, à la découverte de la spécificité sonore des musiques extra-européennes, par exemple. II est parallèle à la découverte, dans la musique contemporaine, d'une splendeur sonore qui elle aussi, à sa manière, dépasse le standard de l'orchestre symphonique. Et par conséquent, je crois que c'est un phénomène qui fait partie d'un mouvement beaucoup plus large qui est une nouvelle écoute de la musique » (Beaussant, 2001, p. 115).

\section{ET AUJOURD'HUI ?}

Il est clair aujourd'hui pour tout le monde que la minorité active des «baroqueux » timbrés a gagné : les ensembles de musique ancienne jouant sur instruments d'époque ou sur copies ont envahi le paysage musical et s'imposent crânement dans les festivals internationaux d'opéras les plus huppés comme celui de Salzbourg ou d'Aix en Provence pour accompagner les opéras de Monteverdi, Charpentier, Haendelou Mozart. De leur côté, les jeunes instrumentistes choisissent de jouer sur l'instrument qui convient le mieux au répertoire interprété passant d'un violon « baroque » monté avec des cordes en boyaux mises en vibration par un archet spécifique, pour l'interprétation des œuvres pré-classiques, à un violon moderne. Les grands orchestres symphoniques jouant sur instruments modernes ne se risquent que peu à interpréter le répertoire baroque et lorsqu'ils le font, c'est sous la baguette de chefs au fait de l'interprétation historiquement informée tels Nikolaus Harnoncourt ou John Elliot Gardiner, qui leur transmettent alors un savoir acquis par ailleurs. Tout iraitil donc pour le mieux dans un monde redevenu «baroque »?

II semble que cela ne soit pas tout à fait le cas. En effet, ça et là s'élèvent des voix regrettant l'institutionnalisation de cette pratique qui s'installerait dans une répétition et pourrait devenir aussi sclérosée et sclérosante que celle contre laquelle elle s'était construite au cours du XXème siècle. Le processus qui s'est passé est le suivant: les timbres qui dérangeaient: contre-ténor, clavecins, instruments à vent naturel et autres flutes en bois ont été apprivoisés par les instrumentistes - l'intonation 
et la justesse n'est plus un problème - et les auditeurs désormais plébiscitent ce qui ce avait pu les gêner ou les rebuter. Le transfert sur l'inouï s'estompe. Pour exemple, les voix de contre-ténors qui avaient pu avec Alfred Deller faire scandale font aujourd'hui recette... Les enregistrements les sollicitant se vendent à des milliers d'exemplaires et Franco Fagioli, le contre-ténor argentin star, au puissant mezzo bien éloigné de la voix fine et éthérée du pionnier Alfred Deller, chante à l'opéra de Paris en 2016 le rôle titre d'un opéra de Cavalli, Eliogabalo, provoque l'enthousiasme des salles de concert et signe un contrat d'enregistrement exclusif chez Deutsch Grammophon qui résistait, à travers Herbert Karajan, à la révolution «baroque » en $1966 . .$.

La quête du son perdu serait-elle achevée et avec elle le principe de plaisir serait-il à nouveau au rendez-vous quand l'inouï le serait de moins en moins ? Cela n'est pas certain. Le transfert sur l'inouï dont je fais l'hypothèse qu'il a orienté le mouvement de redécouverte du répertoire ancien travaille encore la création musicale et c'est encore lui qui fait dresser l'oreille des amateurs.

Pour n'en donner que deux exemples en rapport avec les productions réalisées dans le cadre du Centre Culturel de Rencontres d'Ambronay je citerai tout d'abord l'enthousiasmant enregistrement du Diluvio Universale (créé à Messine en 1682) réalisé par Leonardo Garcia Alarcon en $2011^{18}$. En sollicitant le percussionniste iranien Keyvan Chemirani, dont les improvisations au zarb, oud et tambourin, créent, entre les lamentations des chœurs et les interventions des solistes, des plages de sonorités exotiques, Alarcon déroute l'auditeur le conduisant à entendre d'une façon renouvelée le timbre des voix et des instruments.

Un autre exemple de cette quête toujours active est le remarquable enregistrement du jeune ensemble Prisma ${ }^{19}$ : Les Saisons. Les critiques ne sont s'y pas trompés et ce qui, une fois encore, est mis en avant est « ce vieux désir de bruit » devant lequel les jeunes instrumentistes ne reculent pas. « Ce qui est remarquable avec Prisma, au-delà de leur parfaite connivence musicale et des qualités instrumentales de chacun, c'est leur grande inventivité. Cela s'exprime d'abord dans les petits préludes improvisés : pour le printemps, la flûte d'Elisabeth Champollion

\footnotetext{
${ }^{18}$ // Diluvio universale de Michelangelo Falvetti. Cappella Mediterranea, Chœur de Namur direction : L. García Alarcón. 1 CD Ambronay Éditions 2011.

${ }^{19}$ Les Saisons. Ensemble Prisma. 1 CD Ambronay Éditions 2018.
} 
nous donne à entendre les battements d'ailes des papillons (si, si !); en été, c'est le violon de Franciska Anna Hadju qui se transforme en insecte vrombissant ; à l'automne, le luth d'Alon Sariel évoque la nostalgie de la chute des feuilles; et pour introduire l'hiver, c'est au tour de la viole de David Budai d'évoquer les crissements de la glace ${ }^{20}$. Le crissement, le battement, le vrombissement... autant de termes qui ne concernent pas directement le son musical mais pointe son accointance avec le bruit qui le hante et le fait palpiter. C'est cette palpitation du réel qui continue à orienter les interprètes inspirés et renouvelle, encore et encore, la quête du timbre à jamais perdu et, pourtant, toujours désiré.

${ }^{20}$ Cécile Glaenzer, le 13 décembre 2018, pour le site ResMusica. Disponible em https://www.resmusica.com/2018/12/13/ensemble-prisma-the-seasons-ambronay/ 


\section{RÉFÉRENCES}

BEAUSSANT, P. (1988) Vous avez dit baroque? Arles, Actes Sud. 1994.

BEAUSSANT,P. (2001) «À la recherche du son perdu». Le son des musiques. Entre technologie et esthétique. François Delalande (sous la direction). Paris, INA-Buchet/Chastel.

CHION, M. (1998) Le son. Oü̈r, écouter, observer. Paris, Armand Colin. 2018.

HARNONCOURT, N. (1982) Le Discours musical. Paris, Gallimard. 1984.

HASKELL, H. (1988) Les voix d'un renouveau. La musique ancienne et son interprétation de Mendelssohn à nos jours. Arles, Actes Sud. 2013.

KOECHLIN, C. (1944) Traité d'orchestration. Paris, Max Eschig. 1954.

LANDOWSKA, W. (1909) Renaissance du clavecin au XXème siècle. Musique ancienne. Paris, Bibliothèque des introuvables. 2005.

SALOMOS, M. (2013) De la musique au son. L'émergence du son dans la musique des XXe et XXIe siècles. Rennes, P.U.R. p. 23-85.

SCHAEFFNER, A. (1968) Origine des instruments de musique. Introduction ethnologique à l'histoire de la musique instrumentale. Éditions de l'École des hautes études en sciences sociales, Paris. 1994.

VIVES, J.-M. (2012) La voix sur le divan. Paris, Aubier. 


\section{A Busca Musical Da Autenticidade: Uma TRANSFERÊNCIA DO INAUDITO OU RETRATO DOS “BARROCOSOS" Em Aventureiros Do Timbre Perdido}

\section{RESUMO}

O autor deste artigo se propõe a descrever os fenômenos psíquicos que precederam o movimento barroco em que os músicos buscaram reencontrar os sons que haviam visto nascer as obras interpretadas. Ele se concentra na maneira pela qual o intérprete tenta aproximar um som novo, ainda não advindo, o que o autor chama de "transferência do inaudito". Ele esboça um retrato dos "barrocosos" como aventureiros do timbre perdido. $O$ desejo de autenticidade dos intérpretes seria o de re-escutar timbres que não estavam mais em jogo. $O$ autor aproxima as qualidades deste timbre a partir do paralelo entre André Schaeffner e Philippe Beaussant e, a partir deles, entre as músicas africanas e a aventura barroca: presença do barulho, do trêmulo ou da interferência no som, o que teria o poder de convocar o mais-além do envelope sonoro, a dimensão do real.

PAlAVRAS-ChAVE: barroco, música, timbre, transferência do inaudito, voz. 


\title{
The Quest Of The Authenticity: A Transfer Of The Unheard Or Portrait Of The “Baroquers” In Adventures Of LOST TIMBRE
}

\begin{abstract}
The auteur of this article aims to describe the psychic phenomena which presided the baroque movement where musicians tried to find the sounds that were the ones that have seen the birth of the interpreted works. He focuses on the way the interpreter tries to approach a new sound, not yet heard, which the auteur calls "the transfer of the unheard". He paints a portrait of the "baroquers" as adventures of the lost timbre. The desire for authenticity of the interpreters would be that of re-hearing timbres which were no longer on course. He approaches the qualities of this timbre through the parallel between André Schaeffner and Philippe Beaussant and through them and the African music ant the baroque adventure: the presence of the noise, of the trembling or the trouble in the sound, which could have the power to convoke beyond the sound envelope, the dimension of the reel.
\end{abstract}

KEY-WoRDS: baroque, music, timbre, transfer of the unheard, voice. 
RECEBIDO EM 10/12/2019

APROVADO EM 14/04/2020

(C) 2020 Psicanálise \& Barroco em revista

http://www.seer.unirio.br/index.php/psicanalise-barroco/index revista@psicanaliseebarroco.pro.br

Programa de Pós-Graduação em Memória Social — UNIRIO

Memória, Subjetividade e Criação

www.memoriasocial.pro.br/proposta-area.php 